\title{
Hidden symmetries and the fermionic sector of eleven-dimensional supergravity
}

\author{
Thibault Damour ${ }^{\mathrm{a}}$, Axel Kleinschmidt ${ }^{\mathrm{b}, *}$, Hermann Nicolai ${ }^{\mathrm{b}}$ \\ ${ }^{a}$ Institut des Hautes Etudes Scientifiques, 35, Route de Chartres, F-91440 Bures-sur-Yvette, France \\ ${ }^{\mathrm{b}}$ Max-Planck-Institut für Gravitationsphysik, Albert-Einstein-Institut, Mühlenberg 1, D-14476 Potsdam, Germany \\ Received 14 December 2005; accepted 13 January 2006 \\ Available online 2 February 2006 \\ Editor: L. Alvarez-Gaumé
}

\begin{abstract}
We study the hidden symmetries of the fermionic sector of $D=11$ supergravity, and the rôle of $K\left(E_{10}\right)$ as a generalised 'R-symmetry'. We find a consistent model of a massless spinning particle on an $E_{10} / K\left(E_{10}\right)$ coset manifold whose dynamics can be mapped onto the fermionic and bosonic dynamics of $D=11$ supergravity in the near space-like singularity limit. This $E_{10}$-invariant superparticle dynamics might provide the basis of a new definition of M-theory, and might describe the 'de-emergence' of spacetime near a cosmological singularity.
\end{abstract}

(c) 2006 Elsevier B.V. All rights reserved.

Eleven-dimensional supergravity $\left(\mathrm{SUGRA}_{11}\right)[1]$ is believed to be the low-energy limit of the elusive 'M-theory', which is, hopefully, a unified framework encompassing the various known string theories. Understanding the symmetries of SUGRA $_{11}$ is therefore important for reaching a satisfactory formulation of M-theory. Many years ago it was found that the toroidal dimensional reduction of SUGRA 11 to lower dimensions leads to the emergence of unexpected ('hidden') symmetry groups, notably $E_{7}$ in the reduction to four noncompactified spacetime dimensions [2], $E_{8}$ in the reduction to $D=3$ [2-5], and the affine Kac-Moody group $E_{9}$ in the reduction to $D=2$ [6,7]. It was also conjectured [8] that the hyperbolic Kac-Moody group $E_{10}$ might appear when reducing SUGRA 11 to only one (time-like) dimension.

Recently, the consideration, à la Belinskii, Khalatnikov and Lifshitz [9], of the near space-like singularity limit ${ }^{1}$ of generic inhomogeneous bosonic eleven-dimensional supergravity solutions has uncovered some striking evidence for the hidden rôle of $E_{10}[10,11]$. Ref. [11] related the gradient expansion

\footnotetext{
* Corresponding author.

E-mail address: axel.kleinschmidt@ aei.mpg.de (A. Kleinschmidt).

1 This limit can also be viewed as a small tension limit, $\alpha^{\prime} \rightarrow \infty$.
}

$\left(\partial_{x} \ll \partial_{t}\right)$, which organises the near space-like singularity limit [12], to an algebraic expansion in the height of positive roots of $E_{10}$. A main conjecture of [11] was the existence of a correspondence between the time evolution, around any given spatial point $\mathbf{x}$, of the supergravity bosonic fields $g_{M N}^{(11)}(t, \mathbf{x})$, $A_{M N P}^{(11)}(t, \mathbf{x})$, together with their infinite towers of spatial gradients, on the one hand, and the dynamics of a structureless massless particle on the infinite-dimensional coset space $E_{10} / K\left(E_{10}\right)$ on the other hand. Here, $K\left(E_{10}\right)$ is the maximal compact subgroup of $E_{10}$. Further evidence for the rôle of the one-dimensional non-linear sigma model $E_{10} / K\left(E_{10}\right)$ in M-theory was provided in [13-16].

An earlier and conceptually different proposal aiming at capturing hidden symmetries of M-theory, and based on the very-extended Kac-Moody group $E_{11}$, was made in $[17,18]$ and further developed in [19-21]. A proposal combining the ideas of [18] and [11] was put forward in [22-24].

In this Letter, we extend the bosonic coset construction of [11] to the full supergravity theory by including fermionic variables; more specifically, we provide evidence for the existence of a correspondence between the time evolution of the coupled supergravity fields $g_{M N}^{(11)}(t, \mathbf{x}), A_{M N P}^{(11)}(t, \mathbf{x}), \psi_{M}^{(11)}(t, \mathbf{x})$ and the dynamics of a spinning massless particle on $E_{10} / K\left(E_{10}\right)$. Pre- 
vious work on $E_{10}$ which included fermions can be found in $[13,25] .^{2}$

To motivate our construction of a fermionic extension of the bosonic one-dimensional $E_{10} / K\left(E_{10}\right)$ coset model we consider the equation of motion of the gravitino in $D=11$ supergravity [1]. ${ }^{3}$ Projecting all coordinate indices on an elfbein $E_{(11)}^{A}=$ $E_{(11) M}^{A} d x^{M}$, the equation of motion for $\psi_{A}^{(11)}=E_{(11) A}^{M} \psi_{M}^{(11)}$ is (neglecting quartic fermion terms)

$0=\hat{\mathcal{E}}_{A}:=\Gamma^{B}\left[\left(D_{A}(\omega)+\mathcal{F}_{A}\right) \psi_{B}^{(11)}-\left(D_{B}(\omega)+\mathcal{F}_{B}\right) \psi_{A}^{(11)}\right]$,

where $D_{A}(\omega)=E_{(11) A}^{M} D_{M}$ denotes the moving-frame covariant derivative $D_{A}(\omega) \psi_{B}^{(11)}=\partial_{A} \psi_{B}^{(11)}+\omega_{A B C}^{(11)} \psi^{(11) C}+$ $\frac{1}{4} \omega_{A C D}^{(11)} \Gamma^{C D} \psi_{B}^{(11)}$, and where $\mathcal{F}_{A}:=+\frac{1}{144}\left(\Gamma_{A} B C D E\right.$ $\left.-8 \delta_{A}^{B} \Gamma^{C D E}\right) F_{B C D E}^{(11)}$ denotes the terms depending on the 4form field strength $F_{M N P Q}^{(11)}=4 \partial_{[M} A_{N P Q]}^{(11)}$. Here $\omega_{A B C}^{(11)}=$ $-\omega_{A C B}^{(11)}=E_{(11) A}^{M} \omega_{M B C}^{(11)}$ denotes the moving frame components of the spin connection, with $\omega_{A B C}^{(11)}=\frac{1}{2}\left(\Omega_{A B C}^{(11)}+\right.$ $\Omega_{C A B}^{(11)}-\Omega_{B C A}^{(11)}$, where $\Omega_{A B C}^{(11)}=-\Omega_{B A C}^{(11)}$ are the coefficients of anholonomicity. Following $[11,15]$ we use a pseudoGaussian (zero-shift) coordinate system $t, x^{m}$ and we accordingly decompose the elfbein $E_{(11)}^{A}$ in separate time and space parts as $E_{(11)}^{0}=N d t, E_{(11)}^{a}=e_{(10) m}^{a} d x^{m}$. We note that the zehnbein $E_{(11)}^{a}=e_{(10)}^{a}$ is related to the non-orthogonal, timeindependent spatial frame $\theta^{i}(\mathbf{x})=\theta^{i}{ }_{m}(\mathbf{x}) d x^{m}$ used in [11] via $e_{(10)}^{a}=S^{a}{ }_{i} \theta^{i}[15]$.

Using the $D=11$ local supersymmetry to impose the relation $\psi_{0}^{(11)}=\Gamma_{0} \Gamma^{a} \psi_{a}^{(11)}$, and defining $\mathcal{E}_{a}:=N g^{1 / 4} \Gamma^{0} \hat{\mathcal{E}}_{a}$ (with $\left.g^{1 / 2}=\operatorname{det}\left(e_{(10) m}^{a}\right)\right)$, we find that the spatial components of the gravitino equation of motion (1), when expressed in terms of a rescaled $\psi_{a}^{(10)}:=g^{1 / 4} \psi_{a}^{(11)}$, take the following form

$$
\begin{aligned}
\mathcal{E}_{a}= & \partial_{t} \psi_{a}^{(10)}+\omega_{t a b}^{(11)} \psi^{(10) b}+\frac{1}{4} \omega_{t c d}^{(11)} \Gamma^{c d} \psi_{a}^{(10)} \\
& -\frac{1}{12} F_{t b c d}^{(11)} \Gamma^{b c d} \psi_{a}^{(10)}-\frac{2}{3} F_{t a b c}^{(11)} \Gamma^{b} \psi^{(10) c} \\
& +\frac{1}{6} F_{t b c d}^{(11)} \Gamma_{a}^{b c} \psi^{(10) d}+\frac{N}{144} F_{b c d e}^{(11)} \Gamma^{0} \Gamma^{b c d e} \psi_{a}^{(10)} \\
& +\frac{N}{9} F_{a b c d}^{(11)} \Gamma^{0} \Gamma^{b c d e} \psi_{e}^{(10)}-\frac{N}{72} F_{b c d e}^{(11)} \Gamma^{0} \Gamma_{a b c d e f} \psi^{(10) f} \\
& +N\left(\omega_{a b c}^{(11)}-\omega_{b a c}^{(11)}\right) \Gamma^{0} \Gamma^{b} \psi^{(10) c}+\frac{N}{2} \omega_{a b c}^{(11)} \Gamma^{0} \Gamma^{b c d} \psi_{d}^{(10)}
\end{aligned}
$$

\footnotetext{
2 Results similar to some of the ones reported here have been obtained in [26].

3 We use the mostly plus signature; $M, N, \ldots=0, \ldots, 10$ denote spacetime coordinate (world) indices; $m, n, p, \ldots=1, \ldots, 10$ denote spatial coordinate indices, and the indices $i, j, k, l=1, \ldots, 10$ label the non-orthonormal frame components $\theta^{i}{ }_{m} d x^{m}$. Spacetime Lorentz (flat) indices are denoted $A, B, C, \ldots, F=0, \ldots, 10$, while $a, b, \ldots, f=1, \ldots, 10$ denote purely spatial Lorentz indices. We use the conventions of $[1,2]$ except for the replacement $\Gamma_{\mathrm{CJS}}^{M}=+i \Gamma_{\text {here }}^{M}$ (linked to the mostly plus signature) which allows us to use real gamma matrices and real (Majorana) spinors. The definition of the Dirac conjugate is $\bar{\psi}:=\psi^{T} \Gamma_{\text {here }}^{0}$, and thus differs from [1] by a factor of $i$. The field strength $F_{M N P Q}^{\text {here }}$ used in this Letter is equal to $+1 / 2$ the one used in [11].
}

$$
\begin{aligned}
& -\frac{N}{4} \omega_{b c d}^{(11)} \Gamma^{0} \Gamma^{b c d} \psi_{a}^{(10)} \\
& +N g^{1 / 4} \Gamma^{0} \Gamma^{b}\left(2 \partial_{a} \psi_{b}^{(11)}-\partial_{b} \psi_{a}^{(11)}-\frac{1}{2} \omega_{c c b}^{(11)} \psi_{a}^{(11)}\right. \\
& \left.-\omega_{00 a}^{(11)} \psi_{b}^{(11)}+\frac{1}{2} \omega_{00 b}^{(11)} \psi_{a}^{(11)}\right)
\end{aligned}
$$

Refs. [11,15] defined a dictionary between the temporalgauge bosonic supergravity fields $g_{m n}^{(11)}(t, \mathbf{x}), A_{m n p}^{(11)}(t, \mathbf{x})$ (and their first spatial gradients: spatial connection and magnetic 4-form) and the four lowest levels $h_{a}^{i}(t), A_{i j k}(t), A_{i_{1} \ldots i_{6}}(t)$, $A_{i_{0} \mid i_{1} \ldots i_{8}}(t)$ of the infinite tower of coordinates parametrising the coset manifold $E_{10} / K\left(E_{10}\right)$. Here, we extend this dictionary to fermionic variables by showing that the rescaled, SUSY gauge-fixed gravitino field $\psi_{a}^{(10)}$ can be identified with the first rung of a 'vector-spinor-type' representation of $K\left(E_{10}\right)$, whose Grassmann-valued representation vector will be denoted by $\Psi=\left(\psi_{a}, \psi_{\ldots}, \ldots\right){ }^{4}$ We envisage $\Psi$ to be an infinitedimensional representation of $K\left(E_{10}\right)$ which is decomposed into a tower of $S O(10)$ representations, starting with a vectorspinor one $\psi_{a}$. Our labelling convention is that coset quantities, such as $A_{i j k}$ or $\Psi$ do not carry sub- or superscripts, whereas supergravity quantities carry an explicit dimension label.

We shall give several pieces of evidence in favour of this identification and of the consistency of this $K\left(E_{10}\right)$ representation. As in the bosonic case, the correspondence $\psi_{a}^{(10)}(t, \mathbf{x}) \leftrightarrow$ $\psi_{a}^{\operatorname{coset}}(t) \equiv \psi_{a}(t)$ is defined at a fixed, but arbitrary, spatial point $\mathbf{x}$. A dynamical system governing a 'massless spinning particle' on $E_{10} / K\left(E_{10}\right)$ will be presented as an extension of the coset dynamics of [11] and we will demonstrate the consistency of this dynamical system with the supergravity model under this correspondence. More precisely, we will first show how to consistently identify the Rarita-Schwinger equation (2) with a $K\left(E_{10}\right)$-covariant equation

$0=\stackrel{\text { vs }}{\mathcal{D}} \Psi(t):=\left(\partial_{t}-\stackrel{\text { vs }}{\mathcal{Q}}(t)\right) \Psi(t)$

This equation expresses the parallel propagation of the vectorspinor-type ' $K\left(E_{10}\right)$ polarisation' $\Psi(t)$ along the $E_{10} / K\left(E_{10}\right)$ worldline of the coset particle. Our notation here is as follows. A one-parameter dependent generic group element of $E_{10}$ is denoted by $\mathcal{V}(t)$. The Lie algebra valued 'velocity' of $\mathcal{V}(t)$, namely $v(t)=\partial_{t} \mathcal{V} \mathcal{V}^{-1} \in \mathfrak{e}_{10} \equiv \operatorname{Lie}\left(E_{10}\right)$ is decomposed into its 'symmetric' and 'antisymmetric' parts according to $\mathcal{P}(t):=v_{\mathrm{sym}}(t):=\frac{1}{2}\left(v(t)+v^{T}(t)\right), \mathcal{Q}(t):=v_{\mathrm{anti}}(t):=$ $\frac{1}{2}\left(v(t)-v^{T}(t)\right)$, where the transposition $(\cdot)^{T}$ is the generalised transpose of an $\mathfrak{e}_{10}$ Lie algebra element $x^{T}:=-\omega(x)$ defined by the Chevalley involution $\omega$ [27]. $K\left(E_{10}\right)$ is defined as the set of 'orthogonal elements' $k^{-1}=k^{T}$. Its Lie algebra $\mathfrak{k}_{10}=\operatorname{Lie}\left(K\left(E_{10}\right)\right)$ is made of all the antisymmetric elements of $\mathfrak{e}_{10}$, such as $\mathcal{Q}$.

The bosonic coset model of [11] is invariant under a global $E_{10}$ right action and a local $K\left(E_{10}\right)$ left action $\mathcal{V}(t) \rightarrow$ $k(t) \mathcal{V}(t) g_{0}$. Under the local $K\left(E_{10}\right)$ action, $\mathcal{P}$ varies covari-

\footnotetext{
${ }^{4}$ By contrast, [25] considered 'Dirac-spinor-type' representations of $K\left(E_{10}\right)$.
} 
antly as $\mathcal{P} \rightarrow k \mathcal{P} k^{-1}$, while $\mathcal{Q}$ varies as a $K\left(E_{10}\right)$ connection $\mathcal{Q} \rightarrow k \mathcal{Q} k^{-1}+\partial_{t} k k^{-1}$, with $\partial_{t} k k^{-1} \in \mathfrak{k}_{10}$ following from the orthogonality condition. The coset equation (3) will therefore be $K\left(E_{10}\right)$ covariant if $\Psi$ varies, under a local $K\left(E_{10}\right)$ left action, as a certain ('vector-spinor') linear representation

$\Psi \rightarrow \stackrel{\mathrm{vs}}{R}(k) \cdot \Psi$

and if $\stackrel{\text { vs }}{\mathcal{Q}}$ in (3) is the value of $\mathcal{Q} \in \mathfrak{k}_{10}$ in the same representation $\stackrel{\text { vs }}{R}$. In order to determine the concrete form of $\stackrel{\text { vs }}{\mathcal{Q}}$ in the vector-spinor representation we need an explicit parametrisation of the coset manifold $E_{10} / K\left(E_{10}\right)$.

Following $[11,15]$ we decompose the $E_{10}$ group w.r.t. its $G L(10)$ subgroup. Then the $\ell=0$ generators of $\mathfrak{e}_{10}$ are $\mathfrak{g l}(10)$ generators $K^{a}{ }_{b}$ satisfying the standard commutation relations $\left[K^{a}{ }_{b}, K^{c}{ }_{d}\right]=\delta_{b}^{c} K^{a}{ }_{d}-\delta_{d}^{a} K^{c}{ }_{b}$. The $\mathfrak{e}_{10}$ generators at levels $\ell=$ $1,2,3$ as $G L(10)$ tensors are, respectively, $E^{a_{1} a_{2} a_{3}}=E^{\left[a_{1} a_{2} a_{3}\right]}$, $E^{a_{1} \ldots a_{6}}=E^{\left[a_{1} \ldots a_{6}\right]}$, and $E^{a_{0} \mid a_{1} \ldots a_{8}}=E^{a_{0} \mid\left[a_{1} \ldots a_{8}\right]}$, where the $\ell=3$ generator is also subject to $E^{\left[a_{0} \mid a_{1} \ldots a_{8}\right]}=0$. In a suitable (Borel) gauge, a generic coset element $\mathcal{V} \in E_{10} / K\left(E_{10}\right)$ can be written as $\mathcal{V}=\exp \left(X_{h}\right) \exp \left(X_{A}\right)$ with

$$
\begin{aligned}
X_{h}= & h_{a}^{b} K_{b}^{a}, \\
X_{A}= & \frac{1}{3 !} A_{a_{1} a_{2} a_{3}} E^{a_{1} a_{2} a_{3}}+\frac{1}{6 !} A_{a_{1} \ldots a_{6}} E^{a_{1} \ldots a_{6}} \\
& +\frac{1}{9 !} A_{a_{0} \mid a_{1} \ldots a_{8}} E^{a_{0} \mid a_{1} \ldots a_{8}}+\ldots .
\end{aligned}
$$

Defining $e_{a}^{i}:=(\exp h)^{i}{ }_{a}=\delta^{i}{ }_{a}+h^{i}{ }_{a}+\frac{1}{2 !} h^{i}{ }_{s} h^{s}{ }_{a}+\cdots$ and $\bar{e}^{a}{ }_{i}:=\left(e^{-1}\right)^{a}{ }_{i}$ one finds that the velocity $v \in \mathfrak{e}_{10}$ reads, expanded up to $\ell=3$,

$$
\begin{aligned}
v= & \bar{e}^{b}{ }_{i} \partial_{t} e^{i}{ }_{a} K^{a}{ }_{b}+\frac{1}{3 !} e^{i_{1}} a_{1} e^{i_{2}} a_{2} e^{i_{3}}{ }_{a_{3}} D A_{i_{1} i_{2} i_{3}} E^{a_{1} a_{2} a_{3}} \\
& +\frac{1}{6 !} e^{i_{1}}{ }_{a_{1}} \cdots e^{i_{6}}{ }_{a_{6}} D A_{i_{1} \ldots i_{6}} E^{a_{1} \ldots a_{6}} \\
& +\frac{1}{9 !} e^{i_{0}}{ }_{a_{0}} \cdots e^{i_{8}}{ }_{a_{8}} D A_{i_{0} \mid i_{1} \ldots i_{8}} E^{a_{0} \mid a_{1} \ldots a_{8}}
\end{aligned}
$$

Here, $D A_{i_{1} i_{2} i_{3}}=\partial_{t} A_{i_{1} i_{2} i_{3}}$, and the more complicated expressions for $D A_{i_{1} \ldots i_{6}}$ and $D A_{i_{0} \mid i_{1} \ldots i_{8}}$ were given in [11]. In the expansion (6) of $v$ one can think of the indices on the generators $K_{b}^{a}$, etc., as flat (Euclidean) indices. As for the indices on $D A_{i_{1} i_{2} i_{3}}$, etc., the dictionary of $[11,15]$ shows that they correspond to a time-independent non-orthonormal frame $\theta^{i}=\theta^{i}{ }_{m} d x^{m}$. The object $e^{i}{ }_{a}=(\exp h)^{i}{ }_{a}$ (which is the 'square root' of the contravariant 'coset metric' $\left.g^{i j}=\sum_{a} e_{a}^{i} e^{j}{ }_{a}\right)$ relates the two types of indices, and corresponds to the inverse of the matrix $S^{a}{ }_{i}$ mentioned above. The parametrisation (5) corresponds to a special choice of coordinates on the coset manifold $E_{10} / K\left(E_{10}\right)$.

We introduce the $\mathfrak{k}_{10}$ generators through

$$
\begin{aligned}
& J^{a b}=K_{b}^{a}-K_{a}^{b}, \\
& J^{a_{1} a_{2} a_{3}}=E^{a_{1} a_{2} a_{3}}-F_{a_{1} a_{2} a_{3}}, \\
& J^{a_{1} \ldots a_{6}}=E^{a_{1} \ldots a_{6}}-F_{a_{1} \ldots a_{6}}, \\
& J^{a_{0} \mid a_{1} \ldots a_{8}}=E^{a_{0} \mid a_{1} \ldots a_{8}}-F_{a_{0} \mid a_{1} \ldots a_{8}},
\end{aligned}
$$

where $F_{a_{1} a_{2} a_{3}}=\left(E^{a_{1} a_{2} a_{3}}\right)^{T}$, etc., that is, with the general normalisation $J=E-F$. Henceforth, we shall refer to $J^{a b}, J^{a_{1} a_{2} a_{3}}, J^{a_{1} \ldots a_{6}}$, and $J^{a_{0} \mid a_{1} \ldots a_{8}}$ as being of 'levels' $\ell=$ $0,1,2,3$, respectively. However, this 'level' is not a grading of $\mathfrak{k}_{10}$; rather one finds for commutators that $\left[\mathfrak{k}^{(\ell)}, \mathfrak{k}^{\left(\ell^{\prime}\right)}\right] \subset$ $\mathfrak{k}^{\left(\ell+\ell^{\prime}\right)} \oplus \mathfrak{k}^{\left(\left|\ell-\ell^{\prime}\right|\right)}$ (in fact, $\mathfrak{k}_{10}$ is neither a graded nor a KacMoody algebra). Computing the antisymmetric piece $\mathcal{Q}$ of the velocity $v$ we conclude that the explicit form of the fermionic equation of motion (3) is

$$
\begin{aligned}
& \left(\partial_{t}-\frac{1}{2} \bar{e}^{b}{ }_{i} \partial_{t} e^{i} \underset{a}{\stackrel{\mathrm{vs}}{J}}{ }^{a b}-\frac{1}{2} \frac{1}{3 !} e^{i_{1}} a_{1} \cdots e^{i_{3}}{ }_{a_{3}} D A_{i_{1} \ldots i_{3}} \stackrel{\mathrm{vs}}{J}^{a_{1} a_{2} a_{3}}\right. \\
& -\frac{1}{2} \frac{1}{6 !} e^{i_{1}}{ }_{a_{1}} \cdots e^{i_{6}}{ }_{a_{6}} D A_{i_{1} \ldots i_{6}} J^{\mathrm{vs}_{1} \ldots a_{6}}
\end{aligned}
$$

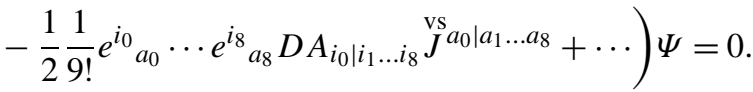

Here, $\stackrel{\text { vs }}{J} a b:=\stackrel{\text { vs }}{R}\left(J^{a b}\right)$, etc., are the form the $\mathfrak{k}_{10}$ generators take in the sought-for vector-spinor representation $\Psi$. The crucial consistency condition for $\Psi$ to be a linear representation is that the generators $\stackrel{\mathrm{vs}}{J}^{a b}$, etc. (to be deduced below) should satisfy the abstract $\mathfrak{k}_{10}$ commutation relations

$$
\begin{aligned}
& {\left[J^{a b}, J^{c d}\right]=\delta^{b c} J^{a d}+\delta^{a d} J^{b c}-\delta^{a c} J^{b d}-\delta^{b d} J^{a c} \equiv 4 \delta^{b c} J^{a d},} \\
& {\left[J^{a_{1} a_{2} a_{3}}, J^{b_{1} b_{2} b_{3}}\right]=J^{a_{1} a_{2} a_{3} b_{1} b_{2} b_{3}}-18 \delta^{a_{1} b_{1}} \delta^{a_{2} b_{2}} J^{a_{3} b_{3}},} \\
& {\left[J^{a_{1} a_{2} a_{3}}, J^{b_{1} \ldots b_{6}}\right]=J^{\left[a_{1} \mid a_{2} a_{3}\right] b_{1} \ldots b_{6}}-5 ! \delta^{a_{1} b_{1}} \delta^{a_{2} b_{2}} \delta^{a_{3} b_{3}} J^{b_{4} b_{5} b_{6}},} \\
& {\left[J^{a_{1} \ldots a_{6}}, J^{b_{1} \ldots b_{6}}\right]=-6 \cdot 6 ! \delta^{a_{1} b_{1}} \ldots \delta^{a_{5} b_{5}} J^{a_{6} b_{6}}+\cdots,} \\
& {\left[J^{a_{1} a_{2} a_{3}}, J^{b_{0} \mid b_{1} \ldots b_{8}}\right]} \\
& \quad=-336\left(\delta_{a_{1} a_{2} a_{3}}^{b_{0} b_{1} b_{2}} J^{b_{3} \ldots b_{8}}-\delta_{a_{1} a_{2} a_{3}}^{b_{1} b_{2} b_{3}} J^{b_{4} \ldots b_{8} b_{0}}\right)+\cdots \\
& {\left[J^{a_{1} \ldots a_{6}}, J^{b_{0} \mid b_{1} \ldots b_{8}}\right]} \\
& \quad=-8 !\left(\delta_{a_{1} \ldots a_{6}}^{b_{0} b_{1} \ldots b_{5}} J^{b_{6} b_{7} b_{8}}-\delta_{a_{1} \ldots a_{6}}^{b_{1} \ldots b_{6}} J^{b_{7} b_{8} b_{0}}\right)+\cdots \\
& {\left[J^{a_{0} \mid a_{1} \ldots a_{8}}, J^{b_{0} \mid b_{1} \ldots b_{8}}\right]} \\
& \quad=-8 \cdot 8 !\left(\delta_{b_{1} \ldots b_{8}}^{a_{1} \ldots a_{8}} J^{a_{0} b_{0}}-\delta_{b_{0} b_{1} \ldots b_{7}}^{a_{1} \ldots a_{8}} J^{a_{0} b_{8}}-\delta_{b_{1} \ldots b_{8}}^{a_{0} a_{1} \ldots a_{7}} J^{a_{8} b_{0}}\right. \\
& \left.\quad+8 \delta_{b_{0}}^{a_{0}} \delta_{b_{1} \ldots b_{7}}^{a_{1} \ldots a_{7}} J^{a_{8} b_{8}}+7 \delta_{b_{0} a_{1}}^{a_{b_{1}} \ldots b_{7}} \delta^{a_{0} a_{2} \ldots a_{7}} J^{a_{8} b_{8}}\right)+\cdots
\end{aligned}
$$

computed up to $\ell=3$ in the basis for $\mathfrak{e}_{10}$ used in [15]. We use the flat Euclidean $\delta^{a b}$ of $S O(10)$ to raise and lower indices. As $S O(10)$ representation the generator $J^{a_{0} \mid a_{1} \ldots a_{8}}$ is reducible with irreducible components $\bar{J}$ and $\hat{J}$ defined by $\bar{J}^{a_{1} \mid a_{2} \ldots a_{9}}=$ $J^{a_{1} \mid a_{2} \ldots a_{9}}-\frac{8}{3} \delta^{a_{1}\left[a_{2}\right.} \hat{J}^{\left.a_{3} \ldots a_{9}\right]}$ and $\hat{J}^{a_{3} \ldots a_{9}}=\delta_{a_{1} a_{2}} J^{a_{1} \mid a_{2} a_{3} \ldots a_{9}} . \mathrm{Ne}-$ glecting $\bar{J}^{a_{0} \mid a_{1} \ldots a_{8}}$, the corresponding commutators for $K\left(E_{11}\right)$ were already computed in [21]. In Eq. (9) we have used a shorthand notation where the terms on the r.h.s. should be antisymmetrised (with weight one) according to the antisymmetries on the 1.h.s., as written out for the $S O(10)$ generators $J^{a b}$ in the first line. For the mixed symmetry generator $J^{a_{0} \mid a_{1} \ldots a_{8}}$ this includes only antisymmetrisation over $\left[a_{1} \cdots a_{8}\right]$. Under $S O(10)$ the tensors on the higher levels rotate in the standard fashion.

To compare Eqs. (2) and (8) we now use the bosonic dictionary obtained in $[11,15]$. In terms of our present conventions, 
and in terms of 'flat' indices on both sides ${ }^{5}$ this dictionary consists of asserting the correspondences

$e^{i}{ }_{a} \leftrightarrow \theta^{i}{ }_{m} e_{(10) a}^{m}$,

$D A_{a_{1} a_{2} a_{3}} \leftrightarrow 2 F_{t a_{1} a_{2} a_{3}}^{(11)}=2 N F_{0 a_{1} a_{2} a_{3}}^{(11)}$,

$D A_{a_{1} \ldots a_{6}} \leftrightarrow-\frac{2}{4 !} N \epsilon_{a_{1} \ldots a_{6} b_{1} \ldots b_{4}} F_{b_{1} \ldots b_{4}}^{(11)}$,

$D A_{a_{0} \mid a_{1} \ldots a_{8}} \leftrightarrow \frac{3}{2} N \epsilon_{a_{1} \ldots a_{8} b_{1} b_{2}} \tilde{\Omega}_{b_{1} b_{2} a_{0}}^{(10)}$.

Here, as in [15], $\tilde{\Omega}_{a b c}^{(10)}=\Omega_{a b c}^{(10)}-\frac{2}{9} \delta_{c[a} \Omega_{b] d d}^{(10)}$ denotes the tracefree part of the spatial anholonomy coefficient $\Omega_{a b c}^{(10)}=$ $2 e_{(10)[a}^{m} e_{(10) b]}^{n} \partial_{m} e_{(10) n}^{c}$.

Using the correspondences (10), as well as their consequence $-\frac{1}{2}\left(\bar{e}^{b}{ }_{i} \partial_{t} e^{i}{ }_{a}-\bar{e}^{a}{ }_{i} \partial_{t} e^{i}{ }_{b}\right) \leftrightarrow+\omega_{t a b}^{(11)}=N \omega_{0 a b}^{(11)}$, we can tentatively reinterpret most terms in the supergravity equation (2) as terms in the putatively $K\left(E_{10}\right)$ covariant equation (8). Using, as is always locally possible, a spatial frame such that the trace $\omega_{b b c}^{(11)}=0$ (and therefore $\tilde{\Omega}_{a b c}^{(10)}=\Omega_{a b c}^{(10)}$ ), and neglecting, as in the bosonic case [11], the frame spatial derivatives $\partial_{a} \psi_{b}^{(10)}$ and $\partial_{a} N=-N \omega_{00 a}^{(11)}$, we can identify Eq. (2) with Eq. (8) if we define the action of $K\left(E_{10}\right)$ generators in the vector-spinor representation by

$$
\begin{aligned}
& \left(\stackrel{\mathrm{Js}}{J}_{\Lambda}^{\mathrm{s}}(0) \Psi\right)_{a}:=\Lambda_{a b} \psi^{b}+\frac{1}{4} \Lambda_{b c} \Gamma^{b c} \psi_{a}, \\
& \left(\stackrel{\mathrm{vs}}{J}_{\Lambda}^{(1)} \Psi\right)_{a} \\
& \quad:=\frac{1}{12} \Lambda_{b c d} \Gamma^{b c d} \psi_{a}+\frac{2}{3} \Lambda_{a b c} \Gamma^{b} \psi^{c}-\frac{1}{6} \Lambda_{b c d} \Gamma_{a b c} \psi^{d}, \\
& \left(\stackrel{\mathrm{v}}{J}_{\Lambda}^{\mathrm{vs}} \Psi\right)_{a} \\
& \quad:=\frac{1}{1440} \Lambda_{b c d e f g} \Gamma^{b c d e f g} \psi_{a}+\frac{1}{180} \Lambda^{b c d e f g} \Gamma_{a b c d e f} \psi_{g} \\
& \quad-\frac{1}{72} \Lambda_{a b c d e f} \Gamma^{b c d e} \psi^{f}, \\
& \left(\stackrel{\mathrm{vs}}{J}_{\Lambda}^{\mathrm{vs}} \Psi\right)_{a} \\
& \quad:=\frac{2}{3} \frac{1}{8 !}\left(\Lambda_{b \mid c_{1} \ldots c_{8}} \Gamma_{a}^{c_{1} \ldots c_{8}} \psi^{b}+8 \Lambda_{a \mid c_{1} \ldots c_{8}} \Gamma^{c_{1} \ldots c c_{7}} \psi^{c_{8}}\right. \\
& \left.\quad+2 \Lambda_{b \mid b c_{1} \ldots c_{7}} \Gamma^{c_{1} \ldots c_{7}} \psi_{a}-28 \Lambda_{b \mid b c_{1} \ldots c_{7}} \Gamma_{a}^{c_{1} \ldots c_{6}} \psi^{c_{7}}\right) .
\end{aligned}
$$

Here, we have used a shorthand notation for the action of $J^{\text {vs }}$ by absorbing the transformation parameters into the generators

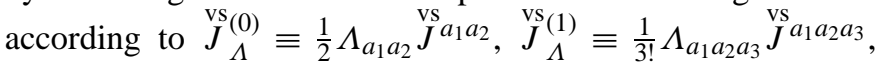

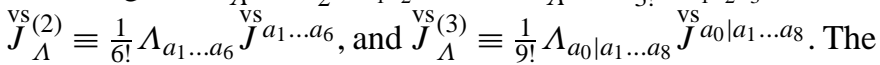
last parameter $\Lambda_{a_{0} \mid a_{1} \ldots a_{8}}$ has two irreducible pieces analogous to $J^{\mathrm{Vs}}(3)$ and the trace appears explicitly in (11).

Proving the $K\left(E_{10}\right)$ covariance of the coset fermionic equation (8) now reduces to proving that the generators $J^{\mathrm{vs}}(\ell)$ defined by (11) do satisfy the $K\left(E_{10}\right)$ commutation relations which were given in (9). It is easy to see that the commutators of the

\footnotetext{
5 To convert 'frame' indices $i, j, k, \ldots$ into 'flat' ones $a, b, c, \ldots$, one uses $e^{i} a$ on the coset side, and $e_{(10) a}^{i}:=\theta^{i}{ }_{m} e_{(10) a}^{m} \equiv\left(S^{-1}\right)^{i} a$ on the SUGRA side.
}

level-zero generators $\stackrel{\mathrm{vs}}{J}^{\mathrm{v}}(0)$ with themselves, as well as with any other $J^{\mathrm{vs}}(\ell)$ for $\ell>0$, produce the required $S O(10)$ rotations of (9). The other commutators require some tedious calculations using the gamma algebra. The result of this computation is

$$
\begin{aligned}
& \left(\left[\stackrel{\mathrm{V}}{J}_{\Lambda}^{\mathrm{v}}(1), \stackrel{\mathrm{vs}}{J}_{\Lambda^{\prime}}^{(1)}\right] \Psi\right)_{a}=20\left(\stackrel{\mathrm{vs}}{J}_{\Sigma}^{(2)} \Psi\right)_{a}-\left(\stackrel{\mathrm{vs}}{J}_{\Sigma}^{(0)} \Psi\right)_{a}, \\
& \left(\left[\stackrel{\mathrm{JS}}{J}_{\Lambda}^{\mathrm{v}}, \stackrel{\mathrm{vs}}{J_{\Lambda}^{\prime}}{ }_{\Lambda^{\prime}}^{(2)}\right] \Psi\right)_{a}=56\left(\stackrel{\mathrm{vs}}{J}_{\Sigma}^{(3)} \Psi\right)_{a}-\frac{1}{6}\left(\stackrel{\mathrm{JS}}{J}_{\Sigma}^{\mathrm{vs}} \Psi\right)_{a},
\end{aligned}
$$

where the $\underset{J}{J} \underset{\Sigma}{\mathrm{vs}(\ell)}$ are defined as above, but now with new parameters given by $\Sigma_{a b}^{(0)}=\Lambda_{d_{1} d_{2}[a} \Lambda_{b]}^{\prime} d_{1} d_{2}, \Sigma_{b_{1} \ldots b_{6}}^{(2)}=\Lambda_{\left[b_{1} b_{2} b_{3}\right.} \times$ $\Lambda_{\left.b_{4} b_{5} b_{6}\right]}^{\prime}, \quad \Sigma_{a_{1} a_{2} a_{3}}^{(1)}=\Lambda^{b_{1} b_{2} b_{3}} \Lambda_{b_{1} b_{2} b_{3} a_{1} a_{2} a_{3}}^{\prime}$, and $\Sigma_{a_{0} \mid a_{1} \ldots a_{8}}^{(3)}=$ $\Lambda_{a_{0}\left[a_{1} a_{2}\right.} \Lambda_{\left.a_{3} \ldots a_{8}\right]}^{\prime}-\Lambda_{\left[a_{1} a_{2} a_{3}\right.} \Lambda_{\left.a_{4} \ldots a_{8}\right] a_{0}}^{\prime}$. One can now check that the relations (12) are consistent with the $K\left(E_{10}\right)$ commutators (9). All other commutators have to produce terms on the r.h.s. which have contributions of 'level' $\ell>3$ and therefore cannot be checked fully. However, we have verified, where possible, that the expected contributions of the lower levels appear with the correct normalisation required by the structure constants of (9). Therefore we find that the vector-spinor representation $J^{\mathrm{J}}(\ell)$ of $K\left(E_{10}\right)$ which we deduced from comparing (2) and (8) is a good linear representation up to the level we have supergravity data to define it.

Using arguments from the general representation theory of Lie algebras one can actually show that the checks we have carried out are sufficient to guarantee the existence of an extension of the vector-spinor representation ${ }^{\mathrm{vs}}(\ell)$ to 'levels' $\ell>3$ on the same components $\psi_{a}$. That is, we can define on $\psi_{a}$ alone an unfaithful, irreducible 320-dimensional representation of $K\left(E_{10}\right)$ on which infinitely many $K\left(E_{10}\right)$ generators are realised non-trivially. For this definition it is sufficient to define the action of $J^{\mathrm{vs}}(0)$ and $J^{\mathrm{vs}}(1)$ on $\psi_{a}$ and check Serre-type compatibility conditions [28]. We view the fact that the $J^{\mathrm{vs}}(2)$ and $J^{\mathrm{Vs}}(3)$ transformations deduced from the supergravity correspondence above agree with this general construction as strong evidence for the relevance of the vector-spinor component of the infinitedimensional $K\left(E_{10}\right)$ spinor $\Psi=\left(\psi_{a}, \ldots\right)$ we have in mind. If one repeats the same analysis for the Dirac spinor, where the representation matrices on this $\mathbf{3 2}$-dimensional space are given in terms of antisymmetric $\Gamma$-matrices (see (16)), one finds that one can consistently realise $K\left(E_{10}\right)$ on a 32-component spinor of $S O(10)$. The fact that the antisymmetric $\Gamma$-matrices together with $\Gamma^{0}$ span the fundamental representation of $S O(32)$ has led a number of authors to propose $S O(32)$ as a 'generalised holonomy' for M-theory [29,30]. That this group, like the larger group $S L(32)$ proposed in [31] cannot be realised as a bona fide symmetry was subsequently pointed out in [32] where it was shown that no suitable spinor (i.e., double valued) representation with the correct number of components of these generalised holonomy groups exist. Our approach is radically different, since we have an action not of $S O(32)$ but of $K\left(E_{10}\right)$, with infinitely many generators acting in a non-trivial manner, on a genuine spinor representation of $S O(10)$. We therefore 
evade the conclusions of [32]. ${ }^{6}$ The appearance of an unfaithful representation for the fermions was already noted and studied in the affine case for $K\left(E_{9}\right)$, which shows very similar features consistent with our present findings [34]. One possibility to construct a faithful representation of $K\left(E_{10}\right)$ already pointed out there might be to consider the tensor product of such unfaithful representations with a faithful representation, like the adjoint $\mathfrak{k}_{10}$ or the coset $\mathfrak{e}_{10} \ominus \mathfrak{k}_{10} .^{7}$ More details on these aspects will be given in a future publication [35].

A deeper confirmation of the hidden $K\left(E_{10}\right)$ symmetry of SUGRA $_{11}$ is obtained by writing down a $K\left(E_{10}\right)$ invariant action functional describing a massless spinning particle on $E_{10} / K\left(E_{10}\right)$. We will be brief and defer the details to [35]. The bosonic part of the action is the one of [11]

$S_{\mathrm{bos}}=\int d t \frac{1}{4 n}\langle\mathcal{P}(t) \mid \mathcal{P}(t)\rangle$,

where $\langle\cdot \mid \cdot\rangle$ is the standard invariant bilinear form on $\mathfrak{e}_{10}$ [27] and where the coset 'lapse' function $n$ can be identified with the rescaled supergravity lapse $N g^{-1 / 2}$ (denoted $\tilde{N}$ in [12]).

The fermionic term we add to this action reads

$S_{\text {ferm }}=-\frac{i}{2} \int d t(\Psi(t) \mid \mathcal{D} \Psi(t))_{\mathrm{vs}}$,

where $(\cdot \mid \cdot)_{\mathrm{vs}}$ is a $K\left(E_{10}\right)$ invariant symmetric form on the vector-spinor representation space. Observe that this symmetric form is actually anti-symmetric when evaluated on anticommuting (Grassmann valued) fermionic variables $\Psi(t)$, such that, e.g., $(\Psi(t) \mid \Psi(t))_{\mathrm{vs}}=0$. On the lowest component of $\Psi=\left(\psi_{a}, \ldots\right)$ it is explicitly given by $(\Psi \mid \Phi)_{\mathrm{vs}}=\psi_{a}^{T} \Gamma^{a b} \phi_{b}$. The invariance of this form under the generators $J^{(\ell)}$ defined in (11) is a quite restrictive condition. We have verified that invariance holds, but only since we are working over a tendimensional Clifford algebra. By using induction arguments we find that $(\Psi \mid \Phi)_{\mathrm{vs}}$ is invariant not only under (11) but under the (unfaithful) extension to the full $K\left(E_{10}\right)$ transformations mentioned above. We expect that the form $(\Psi \mid \Phi)_{\text {vs }}$ will extend to an invariant symmetric form on a faithful representation $\Psi=\left(\psi_{a}, \ldots\right)$.

Further important hints of a hidden $K\left(E_{10}\right)$ symmetry come from considering the local SUSY constraint $\mathcal{S}^{(11)}=0$ which is proportional to the time component of the Rarita-Schwinger equation (1). First, we find that, under the dictionary of [11, $15], \mathcal{S}^{(11)}$ is mapped into a $K\left(E_{10}\right)$ covariant constraint of the form $\mathcal{P} \odot \Psi=0$, when neglecting frame gradients $\partial_{a} \psi_{b}$ as we have done in the derivation of (11). The product $\odot$ symbolises a map from the tensor product of $\mathfrak{e}_{10} \ominus \mathfrak{k}_{10}$ with $\Psi$ onto a Dirac-spinor-type representation space of $\mathfrak{k}_{10}$. The coset constraint $\mathcal{P} \odot \Psi=0$ suggests to augment the action $S_{\text {bos }}+S_{\text {ferm }}$

\footnotetext{
6 The transition from $S O(10)$ to $S O(32)$ (or $S O(1,10)$ to $S L(32)$ ) requires $\Gamma^{a b c}$ which is associated with the rank three gauge field. The importance of the rank three generator in the context of M5-brane dynamics was already stressed in [33] and also features in [21] where it is seen as part of $K\left(E_{11}\right)$. However, it is an open question whether there exists a vector-spinor-type representation of $K\left(E_{11}\right)$, which would be analogous to (11) and thus also compatible with [32].

7 Let us also note that the $\mathbf{3 2 0}$-dimensional representation of $K\left(E_{10}\right)$ is compatible with the fermionic representations studied in [13].
}

by a 'Noether' term of the form

$S_{\text {Noether }}=\int d t(\chi(t) \mid \mathcal{P}(t) \odot \Psi(t))_{\mathrm{s}}$,

with a local Dirac-spinor $\chi(t)$ Lagrange multiplier (that is, a one-dimensional 'gravitino'). The total action $S_{\text {bos }}+S_{\text {ferm }}+$ $S_{\text {Noether }}$ is expected to be not only invariant under $K\left(E_{10}\right)$, but also (disregarding $\Psi^{4}$ terms) under time-dependent supersymmetry transformations which involve a Dirac-spinor-type $K\left(E_{10}\right)$ representation $\epsilon(t)$. In this case the $\chi=0$ gauge fixed action will be invariant under residual quasi-rigid supersymmetry transformations constrained to satisfy $\mathcal{D} \epsilon(t) \equiv$ $\left(\partial_{t}-\stackrel{\mathrm{s}}{\mathcal{Q}}\right) \epsilon=0$. This equation is formally the same as (3) and (8) but now the generators are found to be (cf. [25])

$$
\begin{aligned}
& \stackrel{\mathrm{s}}{J}{ }^{a b}=\frac{1}{2} \Gamma^{a b}, \quad{\stackrel{\mathrm{s}}{J_{1} a_{2} a_{3}}}^{a}=\frac{1}{2} \Gamma^{a_{1} a_{2} a_{3}}, \\
& \stackrel{\mathrm{s}}{J}^{a_{1} \ldots a_{6}}=\frac{1}{2} \Gamma^{a_{1} \ldots a_{6}}, \quad \mathrm{~S}^{a_{0} \mid a_{1} \ldots a_{8}}=12 \delta_{a_{0} b_{1} \ldots b_{7}}^{a_{1} \ldots a_{8}} \Gamma^{b_{1} \ldots b_{7}} .
\end{aligned}
$$

The particular form of the Dirac-spinor representation on $\ell=3$ implies that the irreducible component $\bar{J}^{a_{0}} \mid a_{1} \ldots a_{8}$ is mapped to zero under this correspondence: indeed, there is no way to represent a non-trivial Young tableau purely in terms of gamma matrices. This is in contrast to the vector-spinor representation (11).

In summary, we have given evidence for the following generalisation of the correspondence conjectured in [11]: The time evolution of the eleven-dimensional supergravity fields $g_{M N}^{(11)}(t, \mathbf{x}), A_{M N P}^{(11)}(t, \mathbf{x}), \psi_{M}^{(11)}(t, \mathbf{x})$ and their spatial gradients (considered around any given spatial point $\mathbf{x}$, in temporal gauge and with fixed SUSY gauge) can be mapped onto the dynamics of a (supersymmetric) spinning massless particle $(\mathcal{V}(t), \Psi(t))$ on $E_{10} / K\left(E_{10}\right)$. The $E_{10}$-invariant quantum dynamics of this superparticle might provide the basis of a new definition of M-theory. Much work remains to be done to extend the evidence indicated here, for instance by proving the existence of irreducible faithful (and hence infinite-dimensional) 'vector-spinor-type' and 'Dirac-spinor-type' representations of $K\left(E_{10}\right)$.

Let us finally note on the physical side, that we deem it probable that the proposed correspondence between M-theory and the coset model is such that the two sides do not have a common range of physical validity: indeed, the coset model description emerges in the near space-like singularity limit $T \rightarrow 0$, where $T$ denotes the proper time, ${ }^{8}$ which indicates that the coset description might be well defined only when $T \ll T_{\text {Planck }}$, i.e., in a strong curvature regime where the spacetime description 'deemerges'.

\footnotetext{
8 The coordinate and 'coset time' $t$ used above is (in the gauge $n=1$ ) roughly proportional to $-\log T$, and actually goes to $+\infty$ near the space-like singularity.
} 


\section{Acknowledgements}

We thank Ofer Gabber and Pierre Vanhove for informative discussions and Bernard Julia for clarifying the conventions of $[1,2]$. A.K. and H.N. gratefully acknowledge the hospitality of IHES during several visits. This work was partly supported by the European Research and Training Networks 'Superstrings' (contract number MRTN-CT-2004-512194) and 'Forces Universe' (contract number MRTN-CT-2004-005104).

\section{References}

[1] E. Cremmer, B. Julia, J. Scherk, Phys. Lett. B 76 (1978) 409.

[2] E. Cremmer, B. Julia, Nucl. Phys. B 159 (1979) 141.

[3] B. Julia, Application of supergravity to gravitation theories, in: V. De Sabbata, E. Schmutzer (Eds.), Unified Field Theories of More than 4 Dimensions, World Scientific, Singapore, 1983.

[4] N. Marcus, J.H. Schwarz, Nucl. Phys. B 228 (1983) 145.

[5] H. Nicolai, Phys. Lett. B 187 (1987) 316.

[6] H. Nicolai, Phys. Lett. B 194 (1987) 402.

[7] B. Julia, Group disintegrations, in: M. Roček (Ed.), Superspace and Supergravity, Proceedings of the Nuffield Workshop, Cambridge, 22 June-12 July, 1980, Cambridge Univ. Press, Cambridge, 1981.

[8] B. Julia, in: Lectures in Applied Mathematics, vol. 21, AMS-SIAM, 1985, p. 335, preprint LPTENS 80/16.

[9] V.A. Belinsky, I.M. Khalatnikov, E.M. Lifshitz, Adv. Phys. 19 (1970) 525.

[10] T. Damour, M. Henneaux, Phys. Rev. Lett. 86 (2001) 4749, hepth/0012172.

[11] T. Damour, M. Henneaux, H. Nicolai, Phys. Rev. Lett. 89 (2002) 221601, hep-th/0207267.

[12] T. Damour, M. Henneaux, H. Nicolai, Class. Quantum Grav. 20 (2003) R145, hep-th/0212256.

[13] A. Kleinschmidt, H. Nicolai, JHEP 0407 (2004) 041, hep-th/0407101.
[14] A. Kleinschmidt, H. Nicolai, Phys. Lett. B 606 (2005) 391, hep-th/ 0411225 .

[15] T. Damour, H. Nicolai, Eleven-dimensional supergravity and the $E_{10} / K\left(E_{10}\right) \sigma$-model at low $A_{9}$ levels, in: Group Theoretical Methods in Physics, in: Institute of Physics Conference Series, vol. 185, IOP Publishing, 2005, hep-th/0410245.

[16] T. Damour, H. Nicolai, Class. Quantum Grav. 22 (2005) 2849, hep-th/ 0504153.

[17] P.C. West, JHEP 0008 (2000) 007, hep-th/0005270.

[18] P.C. West, Class. Quantum Grav. 18 (2001) 4443, hep-th/ 0104081.

[19] I. Schnakenburg, P.C. West, Phys. Lett. B 517 (2001) 421, hep-th/ 0107181.

[20] I. Schnakenburg, P.C. West, Phys. Lett. B 540 (2002) 137, hep-th/ 0204207.

[21] P.C. West, Phys. Lett. B 575 (2003) 333, hep-th/0307098.

[22] F. Englert, L. Houart, JHEP 0401 (2004) 002, hep-th/0311255.

[23] F. Englert, L. Houart, JHEP 0405 (2004) 059, hep-th/0405082.

[24] F. Englert, M. Henneaux, L. Houart, JHEP 0502 (2005) 070, hepth/0412184.

[25] S. de Buyl, M. Henneaux, L. Paulot, Class. Quantum Grav. 22 (2005) 3595, hep-th/0506009.

[26] S. de Buyl, M. Henneaux, L. Paulot, hep-th/0512292.

[27] V.G. Kac, Infinite-Dimensional Lie Algebras, third ed., Cambridge Univ. Press, Cambridge, 1990.

[28] S. Berman, Commun. Alg. 17 (1989) 3165.

[29] M.J. Duff, K.S. Stelle, Phys. Lett. B 253 (1991) 113.

[30] M. Duff, J.T. Liu, Nucl. Phys. B 674 (2003) 217, hep-th/0303140.

[31] C. Hull, hep-th/0305039.

[32] A. Keurentjes, Class. Quantum Grav. 21 (2004) 1695, hep-th/0309106.

[33] O. Bärwald, P.C. West, Phys. Lett. B 476 (2000) 157, hep-th/9912226.

[34] H. Nicolai, H. Samtleben, Q.J. Pure Appl. Math. 1 (2005) 180, hepth/0407055.

[35] T. Damour, A. Kleinschmidt, H. Nicolai, in preparation. 